\title{
Determinación de los niveles de cortisol salival en una muestra de sujetos de Santiago de Chile
}

\author{
MACARENA LÉPEZ ${ }^{1 \mathrm{a}}$, EGARDO CAAMAÑO ${ }^{1 \mathrm{a}}$, CARMEN ROMERO $^{\text {tb }}$, \\ JENNY FIEDLER ${ }^{2 b}$, VERÓNICA ARAYA ${ }^{3}$
}

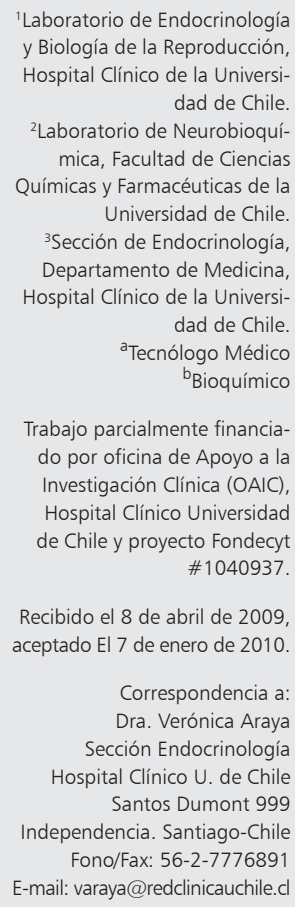

\section{Salivary cortisol among subjects with and without Cushing syndrome}

\begin{abstract}
Background: Salivary cortisol measurement is recommended as a screening measure when a Cushing Syndrome is suspected. The proposed cut-off point for a probable diagnosis is $0.16 \mathrm{ug} / \mathrm{dL}$. Aim: To determine salivary cortisol concentrations during the day in patients with and without Cushing syndrome and with depression. Material and Methods: Salivary cortisol was measured by competitive enzyme immuno assay (EIA), in samples obtained at 8:00, 15:00 and 23:00 h in 78 patients without Cushing syndrome, aged $40 \pm 15$ years (28 males), 30 patients with depression aged $40 \pm 12$ years (nine males) and four females with Cushing syndrome aged $42 \pm 17$ years. Results: Salivary cortisol was higher among patients with Cushing syndrome than the rest of patients. A salivary cortisol over the cut-off value of $0.16 \mathrm{ug} / \mathrm{dL}$ was found in $42 \%$ of subjects without Cushing syndrome and in 33\% of patients with depression. Median values among patients without Cushing syndrome, depression and with Cushing syndrome were 0.21 (range $<0.1-1.42), 0.2$ (range 0,12-0.9) and 0.58 (range 0.37-1.1) ug/dL, respectively. Conclusions: Salivary cortisol measured by EIA method was higher among patients with Cushing syndrome but there was a great overlap with values obtained in subjects without the syndrome.
\end{abstract}

(Rev Med Chile 2010; 138: 168-174).

Key words: Cushing syndrome; Hydrocortisone; Saliva.
L a medición del cortisol nocturno es un buen examen para diagnosticar el síndrome de Cushing (SC), patología en la que se pierde el ritmo circadiano, observándose valores elevados de cortisol a las 23:00 horas, a diferencia de los normales en que la concentración más elevada se alcanza al despertarse ${ }^{1,2}$. La evaluación del cortisol sérico nocturno es difícil de realizar por el inconveniente que implica una punción venosa en este horario.

Por esto, la determinación del cortisol libre en saliva (Sal-cor) ofrece ventajas sobre la medición en suero ${ }^{3-10}$. Algunos estudios han establecido que, en pacientes con SC, la concentración de cortisol libre en saliva tiene una buena correlación con el medido en orina ${ }^{6,11,12}$. La toma de muestra se facilita porque puede recolectarse en la casa, no es invasivo, es indoloro y no produce estrés. Además, el cortisol se mantiene estable por varias semanas en la saliva ${ }^{3,8,13}$.

El algoritmo actual para el diagnóstico de SC incorporó la medición del Sal-cor de 23:00 hrs (Sal-cor23:00) como método de screening ${ }^{14,15}$ ya que tiene una sensibilidad de 60 a $100 \%$ y una especificidad de 70 a 100\% para el diagnóstico en adultos $^{4-7,9-12,16}$.

Algunos autores han propuesto que un valor sobre $0,31 \mu \mathrm{g} / \mathrm{dl}(8,6 \mathrm{nmol} / \mathrm{L})$ hace altamente pro- 
bable el diagnóstico de SC y que un valor menor a $0,16 \mu \mathrm{g} / \mathrm{dl}(4,3 \mathrm{nmol} / \mathrm{L})$ lo hace improbable ${ }^{14,17}$. Sin embargo, existen diferencias en los valores publicados que dependen del ensayo utilizado y de las características de los sujetos analizados. En la mayoría de los estudios se ha utilizado el radio inmunoanálisis (RIA) $)^{6,9-12}$. La técnica de enzimo inmunoanálisis (EIA) ha sido incorporada últimamente en algunos protocolos ${ }^{3,18}$. Otros autores han demostrado que el método de cromatografía líquida con espectrometría de masa en tándem (LC-MS/MS) tendría la ventaja de no tener reactividad cruzada con otros esteroides ${ }^{19}$. Con esta última técnica se ha observado que los sujetos normales tienen un nivel de Sal-cor a la medianoche de $0,14 \mu \mathrm{g} / \mathrm{dl}(4 \mathrm{nmol} / \mathrm{L})$, el que es menor a los obtenidos por RIA y por EIA $^{20}$.

Por otra parte, existen patologías que tienen manifestaciones clínicas o de laboratorio similares al SC, como depresión u obesidad. La determinación del Sal-cor23:00 puede ser útil en el diagnóstico diferencial de estos pseudocushing pero es indispensable obtener valores de referencia para cada población.

Por la importancia del cortisol salival en el diagnóstico del SC, el objetivo de este trabajo fue evaluar el ritmo circadiano de cortisol en saliva en una muestra de sujetos adultos de Santiago de Chile sin SC o pseudocushing, utilizando un EIA, para establecer valores de referencia para esta técnica en nuestra población. También se comparó estos valores con los de un grupo de sujetos depresivos y cuatro pacientes con síndrome de Cushing.

\section{Pacientes y Método}

Se reclutó a 78 voluntarios sin SC o pseudocushing aparente (50 mujeres y 28 hombres), entre 18 y 75 años de edad. Los criterios de exclusión fueron: índice de masa corporal (IMC) mayor de $35 \mathrm{~kg} / \mathrm{m}^{2}$, diabetes, insuficiencia hepática o renal crónica, tabaquismo ( $>$ a 3 cigarrillos/día), alcoholismo, embarazo, depresión, uso reciente de ketoconazol, tratamiento con corticoides en el último año o haber cursado con alguna enfermedad aguda en las últimas dos semanas.

También se evaluó a un grupo de 30 pacientes con el diagnóstico de depresión mayor de acuerdo a los criterios DMS IV, realizado por psiquiatra, antes que iniciaran la terapia antidepresiva, y a
4 pacientes de sexo femenino con síndrome de Cushing confirmado, para establecer la existencia de diferencias con el grupo control.

Para cada sujeto se consignó peso y talla y medicamentos utilizados y todos firmaron un consentimiento informado, aprobado por el Comité de Ética del Hospital Clínico de la Universidad de Chile.

Cada sujeto recolectó tres muestras de saliva, a las 8:00, 15:00 y 23:00 horas. Antes de recolectar la saliva se les indicó enjuagar su boca con agua fría, sin cepillarse los dientes. Tampoco debieron comer o beber en la hora previa a la toma de muestra. Se obtuvo aproximadamente $1 \mathrm{ml}$ de saliva, recolectado por expectoración directa dentro de un tubo de vidrio estéril sin aditivos (vacutainer); se les indicó almacenarlas a $4{ }^{\circ} \mathrm{C}$ hasta ser entregadas en el laboratorio. Las muestras se centrifugaron a $2.500 \mathrm{rpm} / 10 \mathrm{~min}$ y se almacenó el sobrenadante a $-20^{\circ} \mathrm{C}$ hasta su procesamiento.

El Sal-cor se determinó mediante un enzimoinmunoensayo competitivo, aprobado por la FDA (DSL-10-671000 ACTIVE ${ }^{\circledR}$ ) y se basa en la competencia entre el cortisol presente en la muestra con un cortisol unido a peroxidasa, por la unión al anticuerpo IgG de conejo específico para cortisol. Este complejo se une a una inmunoglobulina anti IgG inmovilizada en la fase sólida del sistema y posterior a sucesivos lavados, se le adiciona un sustrato cromógeno (tetrametilbenzidina). Este último es oxidado por la peroxidasa a un compuesto coloreado, cuya absorbancia a $450 \mathrm{~nm}$ es indirectamente proporcional a la concentración de cortisol presente en la muestra. La sensibilidad analítica es de $0,1 \mu \mathrm{g} / \mathrm{dl}$ y la especificidad de $100 \%$ para cortisol, aunque presenta una leve reactividad cruzada con otros esteroides, principalmente prednisolona $(58,3 \%)$. La curva estándar de cortisol fue de 0 a 10,0 $\mu \mathrm{g} / \mathrm{dl}$. Las concentraciones de Sal-cor se calcularon por interpolación de la curva estándar de calibrado con el programa MILENIA Data Reduction 3.22. El control de calidad se basó en la incorporación de dos sueros control de concentraciones baja y alta de cortisol. El coeficiente de variación $(\mathrm{CV})$ intraensayo fue de $4,8 \%$ para una dosis de $0,47 \mu \mathrm{g} / \mathrm{dl} ; 2,8 \%$ para $1,41 \mu \mathrm{g} / \mathrm{dl}$ y $1,9 \%$ para $4,09 \mu \mathrm{g} / \mathrm{dl}$. El CV interensayo fue $7,2 \%$ para una dosis de $0,5 \mu \mathrm{g} / \mathrm{dl}, 2,8 \%$ para $1,51 \mu \mathrm{g} / \mathrm{dl}$ y $3,8 \%$ para $4,12 \mu \mathrm{g} / \mathrm{dl}$.

Estadística: los resultados se expresaron como promedio \pm DS. Como el cortisol salival no pre- 
sentó una distribución normal, se presentan los resultados como mediana y rango para los valores $8.00,15.00$ y 23.00 y se utilizaron las pruebas no paramétricas de Wilcoxon y Mann Whitney para comparación entre grupos y de Kruskal Wallis para comparaciones múltiples; para el análisis de correlación se utilizó la prueba de Spearman-rho. Se consideró significativo un valor de $\mathrm{p}<0,05$.

\section{Resultados}

Las características generales de los participantes se presentan en la Tabla 1. Se observa que los sujetos que tenían alguna patología prevalente como hipertensión arterial (HTA), dislipidemia o hipotiroidismo representan un porcentaje bajo en los grupos control y depresivos. Todos ellos se encontraban bajo tratamiento y bien compensados.

En el grupo control no se encontró diferencias significativas para el Sal-cor en los distintos horarios, entre hombres y mujeres o, entre los rangos etáreos: 18-29, 30-49 y > 50 años (Tabla 2).

Como se observa en la Tabla 1, el cortisol de las 8:00, 15:00 y 23:00 h fue significativamente mayor en los casos con SC respecto de los controles y depresivos. No se observó diferencias entre el grupo control y depresivos en ninguno de los horarios.
En los grupos control y depresivos los valores de Sal-cor fueron significativamente más altos a las 8:00, seguido por una marcada disminución a las 15:00 hrs y el valor más bajo se observó a las 23:00 ( $\mathrm{p}<0,0001)$. En las pacientes con SC no se observó diferencias significativas en los valores de cortisol a lo largo del día.

En el grupo control no encontramos correlación entre los niveles de Sal-cor y la edad a ninguno de los horarios evaluados.

Al dividir a los sujetos controles según el IMC: $<30 \mathrm{~kg} / \mathrm{m}^{2}$ y $\geq 30 \mathrm{~kg} / \mathrm{m}^{2}$, como se observa en la Tabla 2, los sujetos con IMC $<30$ tuvieron un cortisol a las 8:00 y 23:00 h significativamente mayor que los sujetos obesos ( $\mathrm{p}=0,0045$ y 0,02 respectivamente). Además, el Sal-cor23:00 mostró una correlación inversa débil con el IMC $(r=-0,3$, $\mathrm{p}=0,009)$. Al realizar el ajuste por el diagnóstico de hipertensión arterial no observamos diferencias en los resultados obtenidos.

La mediana del Sal-cor23:00 fue 0,21 $\mu \mathrm{g} / \mathrm{dl}$ (rango: < 0,1-1,42) en los controles y se observó que la proporción de casos que presentó un valor bajo el rango de detección de la técnica fue similar en los controles y depresivos (39,7\% vs $46,6 \%)$.

La Figura 1 muestra la distribución del Salcor23:00 en los 3 grupos. Considerando el punto de corte de $0,31 \mathrm{mg} / \mathrm{dl}$, definido en la literatura como diagnóstico probable de SC, 15 de los

Tabla 1. Características generales de los de sujetos en los cuales se evaluó el ritmo de cortisol medido en saliva

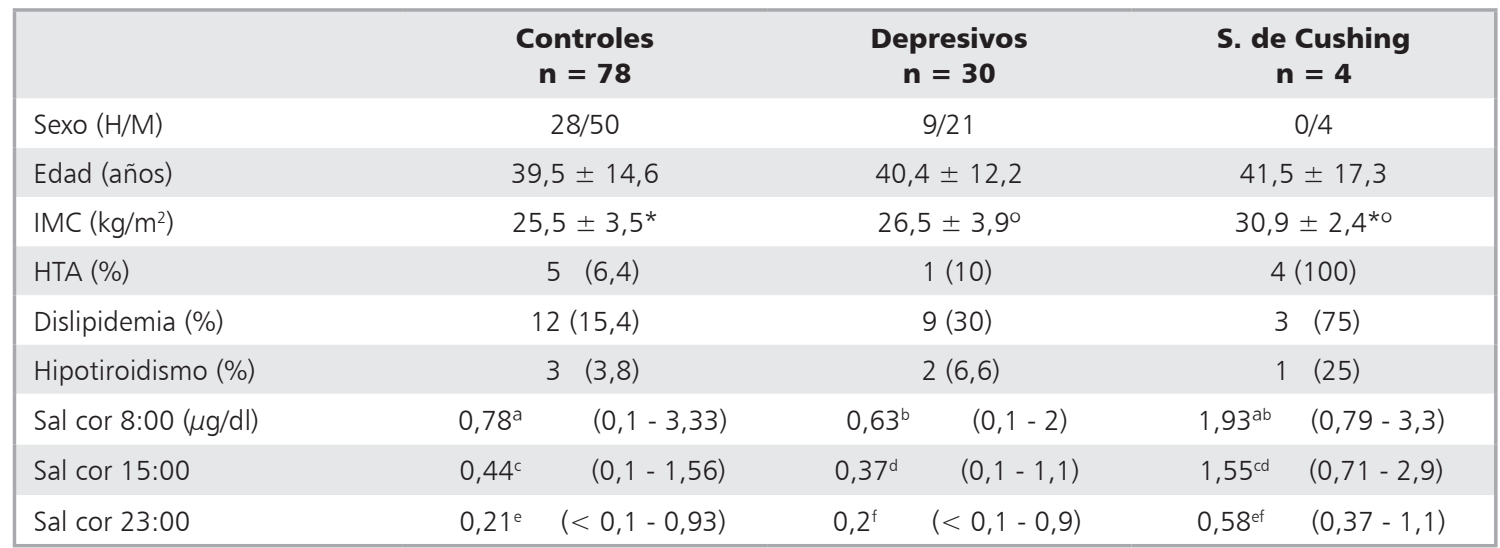

Los valores de cortisol salival se presentan como mediana y rango. Se observó diferencias estadísticamente significativas entre pacientes con síndrome de Cushing vs controles y depresivos. ${ }^{*},{ }^{o} p<0,05 ;{ }^{a} p=0,03 ;{ }^{b} p=0,01 ;{ }^{c, d} p=0,006 ;{ }^{e} p=0,004$; ${ }^{\mathrm{f}} \mathrm{p}=0,005$. 
Tabla 2. Cortisol salival (Sal cor) en $\mu \mathrm{g} / \mathrm{dl}$ en 78 sujetos controles según algunas características clínicas (promedio $\pm D S$ )

\begin{tabular}{|c|c|c|c|}
\hline Casos (n) & Sal cor 8:00 & Sal cor $15: 00$ & Sal cor 23:00 \\
\hline \multicolumn{4}{|l|}{ Edad (años) } \\
\hline $18-29(27)$ & $0,98 \pm 0,6$ & $0,52 \pm 0,2$ & $0,2 \pm 0,2$ \\
\hline $30-49(31)$ & $0,84 \pm 0,68$ & $0,49 \pm 0,32$ & $0,19 \pm 0,12$ \\
\hline$>50(20)$ & $0,82 \pm 0,43$ & $0,47 \pm 0,38$ & $0,29 \pm 0,34$ \\
\hline$P$ & NS & NS & NS \\
\hline \multicolumn{4}{|l|}{ IMC (kg/m²) } \\
\hline$<30(44)$ & $1,04 \pm 0,67$ & $0,54 \pm 0,34$ & $0,24 \pm 0,24$ \\
\hline$>30(34)$ & $0,65 \pm 0,39$ & $0,4 \pm 0,27$ & $0,19 \pm 0,19$ \\
\hline $\mathrm{p}$ & 0,0045 & NS & 0,002 \\
\hline
\end{tabular}

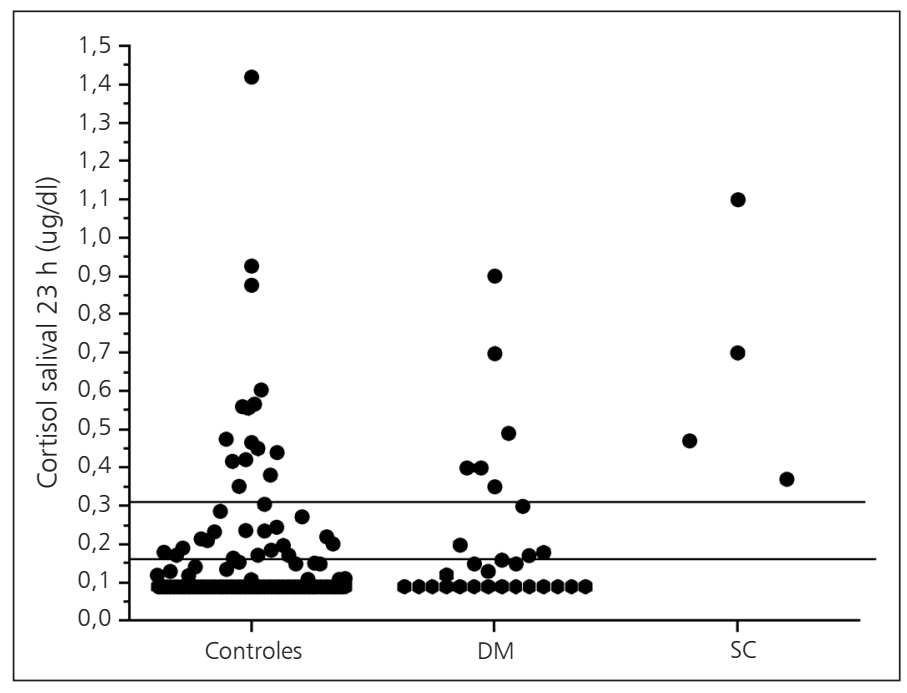

Figura 1. Se observa la distribución del cortisol salival a las 23:00 hrs en un grupo de 78 sujetos controles, 30 pacientes con depresión mayor (DM) y 4 con Síndrome de Cushing (SC), considerando los puntos de corte definidos en la literatura de diagnóstico improbable de SC $(0,16$ $\mu \mathrm{g} / \mathrm{dl})$ y de diagnóstico probable $(0,31 \mu \mathrm{g} / \mathrm{dl})$. controles (19\%) y 6 de los depresivos (20\%) se encontraron sobre este valor. En ellos se evaluó el cortisol libre urinario de 24 hrs y el test de Nugent, descartándose un SC.

Considerando el punto de corte de $0,16 \mu \mathrm{g} /$ dl definido en la literatura como diagnóstico improbable de SC, 33 de los controles (42\%) y 10 de los depresivos (33\%) tuvo un resultado sobre este valor.

\section{Discusión}

La saliva permite estudiar las hormonas esteroidales en su fracción libre, con la ventaja de la fácil obtención de la muestra.
Las guías de práctica clínica americanas para el enfrentamiento de los pacientes con sospecha de SC, proponen la medición del Sal-cor23:00 como una herramienta de primera línea ${ }^{14,15}$. Tendría la ventaja de poder aplicarse en los casos en que la prueba de supresión nocturna con $1 \mathrm{mg}$ de dexametasona o test de Nugent puede verse interferida por problemas de absorción de la dexametasona; aumento de su metabolismo por el uso de algunas drogas como anticonvulsivantes o alcohol o, por disminución del clearence, como en la insuficiencia renal o hepática. Sin embargo, recomiendan que los rangos de normalidad deben ser obtenidos para cada población y de acuerdo a la técnica utilizada. 
Nuestro estudio es el primero en nuestro medio que evalúa el Sal-cor23:00 en una muestra de la población de Santiago de Chile, utilizando un EIA. Esta técnica no produce contaminación radioactiva, es de bajo costo y su sensibilidad para detectar valores bajos de cortisol es superior al método automatizado disponible en nuestro laboratorio. Observamos que la mediana del Salcor23:00 en los controles fue $0,21 \mathrm{mg} / \mathrm{dl}$, valor que está por sobre el propuesto en la literatura internacional como diagnóstico improbable de $\mathrm{SC}^{14,17}$. Esta diferencia puede deberse a la técnica utilizada, ya que en la mayoría de los estudios se usó un RIA y, algunos autores han demostrado que los valores Sal-cor medidos por EIA son más altos, debido a una sobreestimación de la concentración del cortisol por esta técnica ${ }^{19}$. Además, el punto de corte propuesto de $0,16 \mu \mathrm{g} / \mathrm{dl}$ o 4,3 nmol/L proviene del estudio de Raaf, que utilizó un EIA con una sensibilidad similar al RIA, capaz de detectar valores de $0,3 \mathrm{nmol} / \mathrm{L}$ o $0,01 \mu \mathrm{g} / \mathrm{dl}^{21}$. Sin embargo, él evaluó una muestra de 74 sujetos de entre 12 y 84 años, de los cuales, no se detallan las características generales.

Otros factores a considerar son las condiciones de la obtención de la muestra. Se ha demostrado que el tabaquismo, comer o beber alcohol antes de realizarse el examen, contaminación con sangre durante el cepillado dental, enjuagues bucales, realizar actividades psico-estimulantes o el despertarse para la obtención de la muestra, pueden elevar la concentración de Sal-cor ${ }^{22,23}$. Aunque tratamos de controlar estas variables instruyendo a los sujetos en la toma de muestra, no podemos asegurar que fueron cumplidas a cabalidad.

En cuanto al receptáculo utilizado para la recolección de la saliva, en Europa y Estados Unidos de Norteamérica el más recomendado es el Salivette ${ }^{\circledR}$, tubo de propileno que contiene una almohadilla absorbente de fibra sintética que se coloca en la cara interna de la mejilla, evitando el escupir u obtener la muestra por escurrimiento, y para el cual se han publicado buenos resultados en la medición de diferentes analitos ${ }^{24}$. El inconveniente es su elevado costo. También hay que considerar que los artefactos que absorben la saliva y por ende, los compuestos a medir, pueden determinar mediciones erróneamente bajas. La técnica de obtención de la muestra y el tubo que utilizamos nos entregaron resultados reproducibles y consistentes, que hacen improbable una interferencia de los resultados que obtuvimos con el reservorio. Por otra parte, es ampliamente reconocida la gran estabilidad de las hormonas esteroidales frente a diferentes condiciones ambientales. De todas formas, se recomienda en los casos con un resultado alterado, realizar otra determinación antes de continuar el estudio $^{14,15}$.

Otros hechos a considerar son las diferencias étnicas y las características de los sujetos evaluados. Los estudios en población norteamericana han sugerido un aumento del cortisol plasmático nocturno con la edad, fundamentalmente en hombres, lo que podría explicarse por un tiempo de exposición prolongado a situaciones de estrés ${ }^{25}$. Otros autores han reportado elevación del Sal-cor 23:00 en hombres mayores de 60 años, diabéticos e hipertensos ${ }^{18}$. En nuestra muestra, no más de $1 / 3$ de los sujetos tenía más de 50 años Además, excluimos a los sujetos diabéticos o con IMC $>$ de $35 \mathrm{~kg} / \mathrm{m}^{2}$. La proporción de hipertensos fue baja y no hubo diferencias en los resultados al ajustar por esta variable.

Con respecto a la obesidad y niveles de Salcor, Rosmond describió en sujetos con obesidad abdominal, una pobre variación del Sal-cor a lo largo del día, con tendencia a un valor matinal más bajo o un valor vespertino más alto ${ }^{26}$. Esta observación se realizó en hombres suecos con IMC promedio de $27 \mathrm{~kg} / \mathrm{m}^{2}$. Nuestros casos con obesidad, presentaron un cortisol matinal y a las 23:00 h significativamente menor que los no obesos y el IMC se correlacionó en forma inversa con el Sal-cor23:00. Esto podría explicarse por un aumento de la actividad de la enzima $11 \beta$-hidroxi esteroide dehidrogenasa tipo 1 en la grasa visceral, lo que aumentaría la conversión de cortisona a cortisol $^{27,28}$. Este exceso de cortisol generado en el tejido adiposo, puede suprimir al eje corticotropo, determinando valores circulantes más bajos.

Clásicamente se ha descrito que los sujetos depresivos presentan una disfunción del eje suprarrenal que se traduce en un cortisol matinal bajo, cortisol nocturno aumentado y test de Nugent alterado. Nuestros casos no presentaron diferencias con los controles en los horarios evaluados y sólo 2 depresivos tuvieron un Sal-cor23:00 por sobre el promedio de los SC. Esto, probablemente se deba a que los sujetos evaluados en la literatura correspondían en su mayoría a casos con psicosis, hospitalizados por episodios graves, intentos de suicidio, cuadros bipolares o ya tratados con 
múltiples fármacos ${ }^{29,30}$.

En ninguna de las pacientes con SC observamos un valor de Sal-cor23:00 inferior al propuesto como diagnóstico altamente probable $(0,31 \mathrm{mg} / \mathrm{dl}$ o $8,3 \mathrm{nmol} / \mathrm{L}$ ). Aunque es una muestra pequeña, porque la baja incidencia de esta patología dificultó reunir un número mayor de casos durante el período de reclutamiento, y esto impidió realizar una evaluación de sensibilidad, especificidad y valor predictivo para establecer el mejor punto de corte, creemos que estos resultados son significativos y apuntan a que, se debería evaluar cuidadosamente los valores de cortisol salival, de acuerdo a la metodología implementada, antes de aplicar a nuestra población el punto de corte que excluye el diagnóstico de SC establecido por la literatura extranjera $(0,16 \mu \mathrm{g} / \mathrm{dl})$.

En resumen, el cortisol salival es un examen económico y fácil de realizar ante la sospecha de un SC. Sin embargo, demostramos que el nivel de cortisol de 23:00 h medido por técnica de EIA, en una muestra de sujetos adultos sin síndrome de Cushing provenientes de la ciudad de Santiago de Chile, es mayor al publicado en el extranjero para distintos métodos. De acuerdo a nuestros hallazgos, se debería definir un punto de corte para el diagnóstico de screening de SC adecuado a nuestra población y técnicas utilizadas. De esta forma, evitaremos un gasto mayor de recursos en exámenes complementarios.

Agradecimientos: Agradecemos al Sr. Alvaro Reyes por su apoyo en el análisis estadístico de los resultados.

\section{Referencias}

1. Hucklebridge F, Hussain T, Evans P, Clow A. The diurnal patterns of the adrenal steroids cortisol and DHEA in relation to awakening. Psychoneuroendocrinology 2005; 30: 51-7.

2. Kudielka BM, Belligrath S, Hellhammer DH. Further support for higher salivary cortisol levels in "morning" compared to “evening” persons. J Psychosom Res 2007; 62: 595-6.

3. Gozansky WS, Lynn JS, Laudenslager ML, Kohrt WM. Salivary cortisol determinated by enzyme immunoassay is preferable to serum total cortisol for assessment of dynamic hypothalamic-pituitary-adrenal axis activity. Clinical Endocrinology 2005; 63: 336-41.

4. Luthold WW Marcondes JA, Wajchenberg BL. Salivary cortisol for the evaluation of Cushing's syndrome. Clin Chim Acta 1985; 151: 33-9.

5. Laudat MH, Cerdas S, Fournier C, Guiban D, Guilhaume B, Luton JP. Salivary cortisol measurement: a practical approach to assess pituitary-adrenal function. J Clin Endocrinol Metab 1988; 66: 343-8.

6. Raff H, Raff J, Findling JW. Late-night salivary cortisol as a screening test for Cushing's syndrome. J Clin Endocrinol Metab 1998; 83: 2681-26.

7. Castro M, Elias PC, Quidute AR, Halah FP, Moreira AC. Out-patient screening for Cushing's syndrome: the sensitivity of the combination of circadian rhythm and overnight dexamethasone suppression salivary cortisol tests. J Clin Endocrinol Metab 1999; 84: 878-82.

8. Castro M, Elias PC, Martinelli CE Jr, Antonini SR, Santiago L, Moreira AC. Salivary cortisol as a tool for physiological studies and diagnostic strategies. Braz J Med Biol Res. 2000; 33: 1171-5.

9. Papanicolaou DA, Mullen N, Kyrou I, Nieman LK. Nighttime salivary cortisol: a useful test for the diagnosis of Cushing's syndrome. J Clin Endocrinol Metab. 2002; 87: 4515-21.

10. Trilck M, Flitsch J, Lüdecke Dk, Jung R, Petersenn S. Salivary cortisol measurement, a reliable method for the diagnosis of Cushing's syndrome. Exp Clin Endocrinol Diabetes 2005; 113: 225-30.

11. Putignano P, Toja P, Dubini A, Pecori Giraldi F, Corsello SM, Cavagnini F. Midnight salivary cortisol versus urinary free and midnight serum cortisol as screening tests for Cushing's syndrome. J Clin Endocrinol Metab 2003; 88: 4153-7.

12. Viardot A, Huber P, Puder JJ, Zulewski H, Keller U, Müller B. Reproducibility of nighttime salivary cortisol and its use in the diagnosis of hypercortisolism compared with urinary free cortisol and overnight dexamethasone suppression test. J Clin Endocrinol Metab 2005; 90: 5730-6.

13. Poll EM, Kreitschmann-Andermahr I, Langejuergen Y, Stanzel S, Gilsbach JM, Gressner A, et al. Saliva collection method affects predictability of serum cortisol. Clinica Chimica Acta 2007; 382: 15-9.

14. Findling JW, Raff H. Cushing's syndrome: important issues in diagnosis and management. J Clin Endocrinol Metab 2006; 91: 3746-3753.

15. Nieman LK, Biller BMK, Findling JW, Newell-Price J, Savage MO, Stewart PM, et al. The Diagnosis of Cushing's Syndrome: An Endocrine Society Clinical Practice Guideline. J Clin Endocrinol Metab 2008; 93: 1526-40.

16. Yaneva M, Mosnier-Pudar H, Dugué MA, Grabar S, Fulla $\mathrm{Y}$, Bertagna X. Midnight salivary cortisol for the initial 
diagnosis of Cushing's syndrome of various causes. J Clin Endocrinol Metab 2004; 89: 3345-51.

17. Raff H, Finding JW. A physiological approach to diagnosis of Cushing's syndrome. Ann Intern Med 2003; 138: 980-91.

18. Liu H, Bravata DM, Cabaccan J, Raff H, Ryzen E. Elevated late-night salivary cortisol levels in elderly male type 2 diabetic veterans. Clin Endocrinol (Oxf) 2005; 63: 642-9.

19. Raff H, Homar PJ, Burns EA. Comparison of two methods for measuring salivary cortisol [Letter]. Clinical Chemistry 2002; 48: 207-8.

20. Baid SK, Sinaii N, Wade M, Rubino D, Nieman LK. Radioimmunoassay and tandem mass spectrometry measurement of bedtime salivary cortisol levels: a comparison of assays to establish hypercortisolism. J Clin Endocrinol Metab 2007; 92: 3102-7.

21. Raff H, Homar PJ, Skoner DP. New enzyme immunoassay for salivary cortisol [Letter]. Clin Chem 2003; 49: 203-4.

22. Badrick E, Kirschbaum C, Kumasi M. The Relationship between Smoking Status and Cortisol Secretion. J Clin Endocrinol Metab 2007; 92: 819-24.

23. Kivlighan KT, Granger DA, Schwartz EB, Nelson V, Curran M, Shirtcliff EA. Quantifying blood leakage into the oral mucosa and its effects on the measurement of cortisol, dehydroepiandrosterone, and testosterone in saliva. Horm Behav 2004; 46: 39-46.
24. Gröschl M, Köhler H, Topf Hg, Rupprecht T, Rauh M. Evaluation of saliva collection devices for the analysis of steroids, peptides and therapeutic drugs. J Pharm Biomed Anal 2008; 47: 478-86.

25. Van Cauter E, Leproult R, Kupfer DJ. Effects of gender and age on the levels and circadian rhythmicity of plasma cortisol. J Clin Endocrinol Metab. 1996; 81: 2468-73.

26. Rosmond R, Dallman MF, Björntorp P. Stress-Related Cortisol Secretion in Men: Relationships with Abdominal Obesity and Endocrine, Metabolic and Hemodynamic Abnormalities. J Clin Endocrinol Metab. 1998; 83: 1853-9.

27. Stewart PM, Boulton A, Kumar S, Clark PMS, Shackleton CHL. Cortisol metabolism in human obesity: impaired cortisol cortisone conversion in subjects with central adiposity. J Clin Endocrinol Metab 1999; 84: 1022-7.

28. Rask E, Walker BR, Soderberg S, Livingstone DEW, Eliasson M, Johnson O, et al. Tissue-specific changes in peripheral cortisol metabolism in obese women: increased adipose 11b-hydroxysteroid dehydrogenase type 1 activity. J Clin Endocrinol Metab 2002; 87: 3330-6.

29. Nelson, JC, Davis, JM. DST studies in psychotic depression: a meta-analysis. Am. J. Psychiatry 1997; 154: 1497-1503.

30. Keller J, Flores B, Gómez RG, Solvason HB, Kenna H, Williams GH, et al. Cortisol circadian rhythm alterations in psychotic major depression. Biol Psych 2006; 60: 27581. 